\title{
TMEM175 mediates Lysosomal function and participates in neuronal injury induced by cerebral ischemia-reperfusion
}

Mengling Zhang ${ }^{1 \dagger}$, Haifeng $\mathrm{Lu}^{1 \dagger}$, Xueshun $\mathrm{Xie}^{2}$, Haitao Shen ${ }^{2}$, Xiang $\mathrm{Li}^{2}$, Yunhai Zhang ${ }^{3}$, Jiang $\mathrm{Wu}^{2}$, Jianqiang $\mathrm{Ni}^{{ }^{*}}$, Haiying $\mathrm{Li}^{2^{*}}$ (D) and Gang Chen ${ }^{2}$

\begin{abstract}
As the main organelles for the clearance of damaged proteins and damaged organelles, the function of lysosomes is crucial for maintaining the intracellular homeostasis of long-lived neurons. A stable acidic environment is essential for lysosomes to perform their functions. TMEM175 has been identified as a new $\mathrm{K}^{+}$channel that is responsible for regulating lysosomal membrane potential and $\mathrm{pH}$ stability in neurons. This study aimed to understand the role of TMEM175 in lysosomal function of neurons and neuronal injury following cerebral ischemia-reperfusion (I/R). A middle-cerebral-artery occlusion/reperfusion (MCAO/R) model was established in adult male Sprague-Dawley rats in vivo, and cultured neurons were exposed to oxygen-glucose deprivation/reoxygenation (OGD/R) to mimic ischemia-reperfusion (I/R) injury in vitro. We found that the protein level of TMEM175 decreased after cerebral I/R injury and that TMEM175 overexpression ameliorated MCAO/R-induced brain-cell death and neurobehavioral deficits in vivo. Furthermore, these results were recapitulated in cultured neurons. Acridine orange $(A O)$ staining, as well as LysoSensor Green DND-189, cathepsin-B (CTSB), and cathepsin-D (CTSD) activities, showed that TMEM175 deficiency inhibited the hydrolytic function of lysosomes by affecting lysosomal pH. In contrast, TMEM175 upregulation reversed OGD/R-induced lysosomal dysfunction and impaired mitochondrial accumulation in cultured neurons. TMEM175 deficiency induced by cerebral I/R injury leads to compromised lysosomal pH stability, thus inhibiting the hydrolytic function of lysosomes. Consequently, lysosomal-dependent degradation of damaged mitochondria is suppressed and thereby exacerbates brain damage. Exogenous up-regulation of TMEM175 protein level could reverse the neuronal lysosomal dysfunction after ischemia-reperfusion.
\end{abstract}

Keywords: Ischemic stroke, Ischemia-reperfusion injury, Neuron, Lysosome, TMEM175

\section{Introduction}

Cerebral ischemia is a form of stroke that causes considerable disability and mortality worldwide, which induces a heavy burden to both society and affected families. A

\footnotetext{
* Correspondence: njq@suda.edu.cn; Ihy1015@suda.edu.cn

${ }^{\dagger}$ Mengling Zhang and Haifeng Lu contributed equally to this work. 'Department of Neurology, The First Affiliated Hospital of Soochow University, Suzhou, Jiangsu Province, China

2Department of Neurosurgery \& Brain and Nerve Research Laboratory, The First Affiliated Hospital of Soochow University, Suzhou, Jiangsu Province, China

Full list of author information is available at the end of the article
}

brief lack of sufficient blood supply can result in severe brain damage, and reperfusion following ischemia is thought to contribute to delayed secondary brain damage [1-3].

Reperfusion following focal cerebral ischemia leads to cellular changes, including the accumulation of misfolded proteins and organelle damage; in particular, significant mitochondrial dysfunction occurs, such as mitochondrial permeability-transition-pore opening, mitochondrial morphological damage, $\mathrm{Ca}_{2}^{+}$-induced mitochondrial swelling, and the release of mitochondrial 
cytochrome c into the cytosol [4]. Mitochondria have been implicated as central players in the development of ischemic cell death, both through impairment of their normal roles in generating ATP for neuronal function and as key mediators in cell-death pathways [5]. As a result, mitochondrial turnover via the removal of damaged mitochondria is critical to neuronal survival. Recent studies have shown that mitophagy, a lysosomal degradative pathway, is essential for maintaining neuronal homeostasis via the removal of dysfunctional mitochondria following cerebral ischemia-reperfusion (I/R) injury $[6,7]$. Therefore, the hydrolytic function of lysosomes plays a vital role in neuroprotection against ischemic brain injury.

The activities of abundant lysosomal proteases that contribute to the hydrolytic function of lysosomes are inhibited by unstable $\mathrm{pH}$ [8]. Transmembrane protein 175 (TMEM175), recently identified from a lysosomal proteome, is a $\mathrm{K}^{+}$channel located in late endosomes and lysosomes [9]. TMEM175 has been shown to regulate lysosomal membrane potential, $\mathrm{pH}$ stability, and organelle fusion via potassium conductance across lysosomal and endosomal membranes in neurons [9]. TMEM175 deficiency impairs lysosomal pH stability, lysosomemediated autophagosomal clearance, and mitochondrial clearance in neurons [10]. Dysfunctional mitophagy resulting from TMEM175 loss-of-function mutations has been implicated in several human CNS diseases, including Alzheimer's disease [11] and Parkinson's disease [7]. However, the role of TMEM175 in lysosomal hydrolysis and mitochondrial quality control after brain I/ $\mathrm{R}$ injury is unclear. In this study, we aimed to understand the roles of TMEM175 in lysosomal function of neurons and neuronal injury following cerebral I/R.

\section{Materials and methods}

\section{Experimental design}

In experiment 1(Supplementary Figure 1), the levels of TMEM175 and LAMP2 in brain tissues of rats after $\mathrm{MCAO} / \mathrm{R}$ and in primary neurons after $\mathrm{OGD} / \mathrm{R}$ were measured. Forty-two male SD rats were randomly and equally assigned to seven groups of six rats each, as follows: a sham group; and six experimental groups at 1,3 , 6, 12 24, and $48 \mathrm{~h}$ after MCAO/R. At the corresponding time following induction of $\mathrm{MCAO} / \mathrm{R}$, all rats were euthanized by chloral hydrate, and their cerebral tissues were collected for subsequent analysis after transcardial perfusion with $\mathrm{PBS}$. Brain tissues of six rats in each group were extracted and frozen at $-80^{\circ} \mathrm{C}$ until further analysis via Western blotting. Similarly, primary neurons were divided into eight groups as follows: a control group; and OGD/R treatment groups at 1, 3, 6, 12, 24, 48 , and $72 \mathrm{~h}$ after $\mathrm{OGD} / \mathrm{R}$. At the corresponding times, cellular proteins were extracted for Western blot analysis.

In experiment 2(Supplementary Figure 1), the effect of TMEM175 deficiency on neuronal apoptosis and longterm behavior was assessed. Here, 170 male SD rats were randomly divided into four groups of 34 rats each, as follows: (1) sham group;(2) sham + Over-TMEM175 group, in which all rats were administered a TMEM175 plasmid (3) 24-h $\mathrm{MCAO} / \mathrm{R}$ group; (4) $\mathrm{MCAO} / \mathrm{R}+$ vector group, in which all rats were injected with an empty vector in the lateral cerebral ventricle before $48 \mathrm{~h}$ of $\mathrm{MCAO} / \mathrm{R}$ treatment; and (5) MCAO/R + TMEM175-overexpression group, in which all rats were subjected to $\mathrm{MCAO} / \mathrm{R}$ and administered a TMEM175 plasmid (dissolved in Entranster-in vivo DNA transfection reagent) before $48 \mathrm{~h}$ of MCAO/R treatment. Then, the 34 rats in each group except the sham + Over-TMEM175 group were randomly separated into four subgroups via a random-number table. For six rats, total coronal sections containing penumbral brain tissues were harvested and frozen in liquid nitrogen for Western blot analysis. For another six rats, penumbral brain tissues were harvested for Fluoro-Jade C (FJC) staining. Six rats were sacrificed for 2,3,5-Triphenyltetrazolium Chloride (TTC) staining. For behavioral assessments, experimenters were blind to the component of the infusion and the group of rats. Ten rats were trained on the third, fifth, seventh, and fourteen days after surgery in the rotarod test and adhesive-removal test. We trained the last six rats in the Morris Water Maze. For in-vitro experiments, neuronal cultures were randomly divided into the following four groups: (1) control group; (2) control + TMEM175-overexpression group, in which neurons were administered a TMEM175 plasmid (3) 4-h OGD/R group; (4) $\mathrm{OGD} / \mathrm{R}$ + vector group, in which neurons were transfected with an empty vector before $48 \mathrm{~h}$ of OGD/R treatment; and (5) OGD/R + TMEM175-overexpression group, in which neurons were subjected to OGD/R and administered a TMEM175 plasmid (dissolved in Entransterin vivo DNA transfection reagent). Then, neuronal death was assessed via Hoechst-33,258 nuclear staining and a live/dead viability assay.

In experiment 3(Supplementary Figure 1), Four hours after OGD/R, mitochondrial ROS was assessed via MitoSOX dye. Furthermore, assessment of $\Delta \Psi \mathrm{m}$ was detected via JC-1 dye at the same time point. We also investigated the role of TMEM175 loss-of-function mutations on the hydrolytic function of lysosomes in vitro: $\mathrm{AO}$ staining conducted at $2 \mathrm{~h}, 4 \mathrm{~h}$ after $\mathrm{OGD} / \mathrm{R}$ treatment and LysoSensor Green DND-189 conducted at $4 \mathrm{~h}$ after $\mathrm{OGD} / \mathrm{R}$ treatment were used to detect changes in lysosomal $\mathrm{pH}$. The activities of the major lysosomal proteases, CTSB and CTSD, were measured at $4 \mathrm{~h}$ after OGD/R treatment to detect the hydrolytic function of lysosomes. 


\section{Animals}

Animal protocols were approved by the Ethics Committee of the First Affiliated Hospital of Soochow University (Jiangsu Province, China). All animal experiments were in accordance with the National Institute of Health (Bethesda, MD, USA) guidelines for the care and use of experimental animals. Adult male Sprague-Dawley (SD) rats $(300-350 \mathrm{~g})$ were purchased from the Animal Center of the Chinese Academy of Sciences (Shanghai, China). Rats were maintained in a room with controlled temperature $\left(22 \pm 2{ }^{\circ} \mathrm{C}\right)$, relative humidity $(55 \pm 5 \%)$, and a regular light/dark cycle. Food and water were provided ad libitum. We made every effort to reduce the number of animals used and their suffering.

\section{Establishment of experimental middle-cerebral-artery occlusion/reperfusion (MCAO/R) model in rats}

We established a middle-cerebral-artery occlusion/reperfusion model in SD rats, as previously described [12]. Under an operating microscope, the common, internal, and external carotid arteries (CCA, ICA, and ECA, respectively) were exposed through a midline cervical incision. Then, a piece of filament (Xi Nong, Beijing, China) was inserted into the right CCA and advanced along the right ICA until the tip occluded the proximal stem of the middle cerebral artery. After $2 \mathrm{~h}$ of ischemia, the filament was withdrawn for reperfusion. Sham controls only had exposed blood vessels through a midline cervical incision.

\section{Cell cultures}

Primary rat cortical neurons were obtained and cultured as described previously [13]. Pregnant SD rats (16-18 days) were used to prepare primary-neuron-enriched cultures. In brief, we first removed the meninges and blood vessels of the brain. The brain tissues were then digested with $0.25 \%$ trypsin for $5 \mathrm{~min}$, and the digestion was terminated by washing the tissue three times with PBS. The brain tissue suspension was centrifuged at $500 \times \mathrm{g}$ for $5 \mathrm{~min}$, and the pellet was then resuspended in neural basal medium (all from GIBCO, Carlsbad, CA, USA). Finally, cells were seeded in 12-well plates and 6well plates in fresh medium. Afterward, half of the medium was changed every $2 \mathrm{~d}$.

\section{In-vitro application of oxygen-glucose deprivation/ Reoxygenation (OGD/R)}

To mimic I/R in vitro, primary neurons were exposed to oxygen-glucose deprivation/reoxygenation (OGD/R), as previously described [14]. Briefly, the cells were maintained in glucose-free medium in a humidified incubator containing $95 \% \mathrm{~N}_{2}$ and $5 \% \mathrm{CO}_{2}$ at $37^{\circ} \mathrm{C}$ for $2 \mathrm{~h}$. For reoxygenation with glucose reintroduction, cells were again cultured in standard medium and placed in an incubator containing $70 \% \mathrm{~N}_{2}, 25 \% \mathrm{O}_{2}$, and $5 \% \mathrm{CO}_{2}$.

\section{Western blotting}

Western blot analysis was performed as previously reported $[15,16]$. Briefly, the brain samples of rats or harvested neurons in vitro were mechanically lysed in a RIPA lysate buffer (Beyotime, China). The protein concentrations were measured by the bicinchoninic acid (BCA) method using a specific assay kit (Beyotime, China). The protein samples $(30 \mu \mathrm{g} /$ lane $)$ were loaded onto a $10 \%$ SDS-polyacrylamide gel, separated, and then electrophoretically transferred to a polyvinylidenedifluoride (PVDF) membrane (Millipore Corporation, USA). The membrane was blocked with $5 \%$ bovine serum albumin (BSA, BioSharp, China) at $25^{\circ} \mathrm{C}$ for $1 \mathrm{~h}$. Next, the PVDF membrane was incubated with primary antibodies at $4{ }^{\circ} \mathrm{C}$ overnight. The primary antibodies used in this study were as follows: Transmembrane protein 175 (TMEM175) (Proteintech, China, 1:1000 dilution). In addition, $\beta$-tubulin (Cell Signaling Technology, USA) served as a loading control. Finally, the PVDF membrane was incubated with an HRP-conjugated secondary antibody (Cell Signaling Technology, USA) at $25^{\circ} \mathrm{C}$ for $1 \mathrm{~h}$. Protein bands were visualized using an Enhanced Chemiluminescence (ECL) kit (Beyotime, China), and the relative amounts of proteins were analyzed via Image software (NIH, USA) and normalized to the corresponding control sample. In addition, phosphorylation levels were assessed as the ratio of phosphoprotein to total protein.

\section{2,3,5-Triphenyltetrazolium chloride (TTC) staining}

TTC staining was used to detect the infarct volume and was performed as previously reported [17]. Brains were quickly removed, frozen at $-20^{\circ} \mathrm{C}$ for $10 \mathrm{~min}$, and sectioned coronally into 2-mm-thick slices starting from the frontal pole. The olfactory bulb and cerebellum were discarded. Then, the brain slices were immersed in TTC solution (Jiancheng Biotech, Nanjing, China) for 15-30 min at $37^{\circ} \mathrm{C}$. After staining, the brain tissue was differentiated according to the white-colored infarct area and red-purple non-infarct area. Then, these areas of brain tissues were imaged with a digital camera. Cerebral infarct volumes were calculated according to the following formula: (contralateral hemisphere area-(ipsilateral hemisphere area - infarct area)/contralateral hemisphere area) $\times 100 \%$.

\section{Fluoro-jade $\mathrm{C}$ (FJC) staining}

FJC staining was used to evaluate neuronal degeneration. Brain sections were deparaffinized, rehydrated, and incubated in $0.06 \% \mathrm{~K}$ permanganate for $15 \mathrm{~min}$. Then, the brain sections were rinsed in deionized water, immersed 
in $0.001 \%$ FJC working solution ( $0.1 \%$ acetic acid) for 30 min, and dried in an incubator $\left(50-60^{\circ} \mathrm{C}\right)$ for $10 \mathrm{~min}$. The brain sections were cleared in xylene, cover slipped with one drop of mounting medium, and observed under a fluorescent microscope (OLYMPUS BX50/BXFLA/DP70; Olympus Co., Tokyo, Japan).

\section{Cell-death/viability assays}

Four hours after OGD, cell viability and death were quantitatively evaluated by Hoechst-33,258 nuclear staining (Beyotime, China) and a live/dead cell-viability assay (Invitrogen, USA). For Hoechst staining, the percentage of cells showing nuclear condensation was quantified and expressed as a function of the total number of neurons (cell-death percentage). A live/dead cell-viability assay was performed as previously described, according to the manufacturer's instructions. In this assay, red dots (fluorescent ethidium homodimer-1) represent dead cells with compromised membranes, and green dots (fluorescent membrane-permeant calcein AM) represent live cells. For cell counting, three random fields were captured per well by a blinded observer. The experiments were repeated on three independent occasions.

\section{Acridine Orange $(\mathrm{AO})$ staining}

AO staining was used to detect changes in lysosomal $\mathrm{pH}$. In brief, neurons were first rinsed with PBS. Then, cells were stained with AO mix $(10 \mu \mathrm{g} / \mathrm{ml}$ in PBS) for 25 s. Finally, stained neurons were imaged under a confocal microscope (ZEISS LSM 880, Carl Zeiss AG, Germany) from three separate experiments.

\section{Cathepsin-B (CTSB) and Cathepsin-D (CTSD) activities}

CTSB and CTSD activities were measured using commercial assay kits according to the manufacturer's protocols (Biovision, USA). The samples were read in a microplate reader with 400-nm excitation and 505-nm emission for CTSB and 328-nm excitation and 460-nm emission for CTSD.

\section{LysoSensor green DND-189 staining}

Lysosomal staining with the fluorescent acidotropic probe, LysoSensor Green DND-189, was performed according to the manufacturer's recommendations (Invitrogen,USA). LysoSensor Green was then added directly to the medium at a final concentration of $5 \mathrm{mM}$ and incubated for $2 \mathrm{~h}$ at $37^{\circ} \mathrm{C}$, with $5 \% \mathrm{CO} 2$. At the end of the incubation, cells were directly transferred to a fluorescent microscope for imaging.

\section{Measurement of mitochondrial reactive oxygen species} (ROS)

Mitochondrial reactive oxygen species (ROS) in primary cultured neurons were assessed via Mito-Sox staining.
Briefly, neurons were cultured in 12-well plates with glass coverslips and were subjected to different treatments. Next, neurons were incubated with $5-\mu \mathrm{M}$ MitoSox (Invitrogen, USA) for $15 \mathrm{~min}$ at $37^{\circ} \mathrm{C}$ in the dark. Finally, samples were randomly imaged, and their fluorescent intensities were analyzed via ImageJ software from three independent experiments.

\section{Assessment of mitochondrial membrane potential $\left(\Delta \Psi_{m}\right)$}

Primary neurons were stained with the inner mitochondrial-membrane-potential $(\Delta \Psi \mathrm{m})$ reporter dye, JC-1 (BD Bioscience), per the manufacturer's instructions. In brief, JC-1 was prepared to a final concentration of $1 \mu \mathrm{M}$ in diluting buffer, and neurons under different conditions were incubated for $20 \mathrm{~min}$. Stained neurons were examined under a fluorescent microscope. The specific characteristics of $\mathrm{JC}-1$ dye are as follows. When $\Delta \Psi \mathrm{m}$ is low, JC-1 exists as a monomer, and green fluorescence can be detected. By contrast, when $\Delta \Psi \mathrm{m}$ increases, JC-1 assemble into arrays, termed J-aggregates, that exhibit red fluorescence. Therefore, at high $\Delta \Psi \mathrm{m}$, red fluorescence is detected. Fluorescent analysis was carried out by measuring the total fluorescence of the entire oocyte.

\section{Plasmid transfection in vivo and Vitro}

GFP-TMEM175 non-fused overexpressed plasmid and TMEM175 overexpressed plasmid were used to detect the efficiency of plasmid transfection and overexpression. An empty vector was used as a negative control for the TMEM175-overexperssion (Over-TMEM175) construct. Transfection in vivo was performed as described previously [18]. Briefly, $5 \mu \mathrm{g}$ of TMEM175overexpression plasmid was dissolved in $5 \mu \mathrm{l}$ of endotoxin-free water. Then, $10 \mu \mathrm{l}$ of Entranster-in vivo DNA transfection reagent (Engreen Biosystem Co. Lid, $18,688-11-2$ ) was immediately added to $5 \mu \mathrm{l}$ of plasmid. The solution was mixed for $15 \mathrm{~min}$ at room temperature. Finally, $15 \mu \mathrm{l}$ of Entranster-in vivo-plasmid mixture was injected intracerebroventricularly under the guidance of a stereotaxic apparatus after anesthesia. We established the $\mathrm{MCAO} / \mathrm{R}$ model at $24 \mathrm{~h}$ after this process. The puncture point of the lateral ventricle was located at 1.5$\mathrm{mm}$ posterior, $1.0-\mathrm{mm}$ lateral, and $3.2-\mathrm{mm}$ below the horizontal plane of bregma. Cultured neurons were transfected with these two plasmids using Lipofectamine $^{\bullet} 3000$ Transfection Reagent (Invitrogen, L3000015), according to the manufacturer's instructions. At $48 \mathrm{~h}$ after transfection, neurons were treated with OGD/ $\mathrm{R}$.

\section{Morris water maze}

We trained rats 22-25 days after surgery in the Morris water maze. The arena was $50-\mathrm{cm}$ high and $180 \mathrm{~cm}$ in 
diameter and was filled with water to a height of $30 \mathrm{~cm}$ at $20-22^{\circ} \mathrm{C}$. The platform was placed approximately 2 $\mathrm{cm}$ below the water surface. Each trial continued until the rat found the platform and stood on it for $2 \mathrm{~s}$, or until $60 \mathrm{~s}$ had elapsed. After each trial, the rat was placed onto the platform to rest for $20 \mathrm{~s}$. Swimming speeds, as well as latencies and path lengths to reach the platform, were measured as previously described [19]. The behavioral analyses were conducted by an investigator blinded to the treatments.

\section{Rotarod test}

An accelerating rotarod test was employed to measure the motor function of rats [20]. The diameter of the rotarod spindle was $10 \mathrm{~cm}$. The speed of the spindle was increased from 4 to $30 \mathrm{rpm}$ in $60 \mathrm{~s}$, after which $30 \mathrm{rpm}$ was maintained for a maximum of $300 \mathrm{~s}$. When the rats lost their balance and fell off the rotarod, it triggered the sensor, and the time was recorded. Through separation by two panels to prevent the rats from detecting each other, three rats were able to run simultaneously. Before surgery, each rat received three training sessions per day for three consecutive days, and the last three records were counted as the baseline. Then, all rats received a test trial on an accelerating rotarod at all testing days after $\mathrm{MCAO} / \mathrm{R}$.

\section{Adhesive-removal test}

The adhesive removal test was performed as described previously [20]. Briefly, rats were removed from their home cages and were trained to become familiar with the testing environment. Then, two small pieces of adhesive-backed paper dots were glued to the wrist of each forelimb. The rats were then gently returned to their testing cages. The time required to contact and remove both stimuli from each limb was recorded in five trials per day for three days. If the rats were able to remove the dots within $10 \mathrm{~s}$ at the end of training, the rats were included in the experimental group. Then, all rats received a test trial at all testing days after $\mathrm{MCAO} / \mathrm{R}$.

\section{Statistical analysis}

All data are expressed as the mean \pm standard error of the mean (SEM). GraphPad Prism7(GraphPad Software, San Diego, USA) was used for statistical analysis. Data were analyzed by one-way analyses of variance (ANOVAs) to compare differences among multiple groups, and Mann-Whitney $U$ tests were used to analyze nonparametric data. Before the aforementioned tests, datasets in each group were tested for normality via Kolmogorov-Smirnov tests. $P<0.05$ was considered statistically significant (Supplementary Table 1).

\section{Results}

\section{General observations}

No significant differences were observed for body weight, temperature, blood gas, blood glucose, or blood routine-examination data among the experimental groups (data not shown). No animals died (0/40 rats) in the sham group, and the mortality rate of rats was $19.3 \%$ (33/171 rats) after induction of MCAO/R (Supplementary Table 2).

\section{TMEM175 protein levels are decreased in penumbral tissue after ischemia/reperfusion injury}

To investigate whether the protein level of TMEM175 changes after $\mathrm{MCAO} / \mathrm{R}$, we performed $\mathrm{MCAO} / \mathrm{R}$ in SD rats, and Western blot analysis of protein samples from penumbral tissue was subsequently performed. The protein expression level of TMEM175 is normalized by tubulin expression. Compared with that of the sham group, the protein level of TMEM175 was decreased at $3 \mathrm{~h}$ after $\mathrm{MCAO} / \mathrm{R}$ and dropped to its lowest level at 12 $\mathrm{h}$, after which it gradually rebounded (Fig. 1a). In addition, in order to explore the change of protein level of TMEM175 in primary cultured neurons after OGD/R, we performed Western blot analysis on protein samples. Compared with that of the control group, the protein level of TMEM175 decreased at $3 \mathrm{~h}$ after MCAO/R, which is consistent with our results in vivo (Fig. 1b). In order to exclude the possibility that the number of lysosomes decreased after the ischemia/reperfusion injury, the protein level of LAMP2 (lysosomal associated membrane protein 2), a marker for lysosomal and endosomal membranes [21], was investigated in the experiments. We found that both $\mathrm{MCAO} / \mathrm{R}$ treatment of rats and OGD/R treatment of cultured neurons did not decrease the level of LAMP2. The protein expression level of LAMP2 is normalized by GAPDH expression (Fig. 1c-d). These results suggested that the ischemia/reperfusion injury did not impair the quantity of lysosomes.

\section{TMEM175 overexpression improves neuronal death and neurobehavioral deficits after MCAO/R in rats}

To examine the role of TMEM175 deficiency in ischemic injury induced by $\mathrm{MCAO} / \mathrm{R}$, we regulated the protein levels of TMEM175 using over-expression constructs in brain tissues of rats. First, we performed overexpression using plasmid transfection of TMEM175 in our rat model of $\mathrm{MCAO} / \mathrm{R}$. The results of Western blot analysis showed that, compared with that of the sham group, the level of TMEM175 was significantly decreased in the MCAO/R group, while over-expression of TMEM175 increased the level of TMEM175. The protein expression level of TMEM175 is normalized by GAPDH expression. (Fig. 2a). To explore the roles of TMEM175 in neuronal death and degeneration in brain 


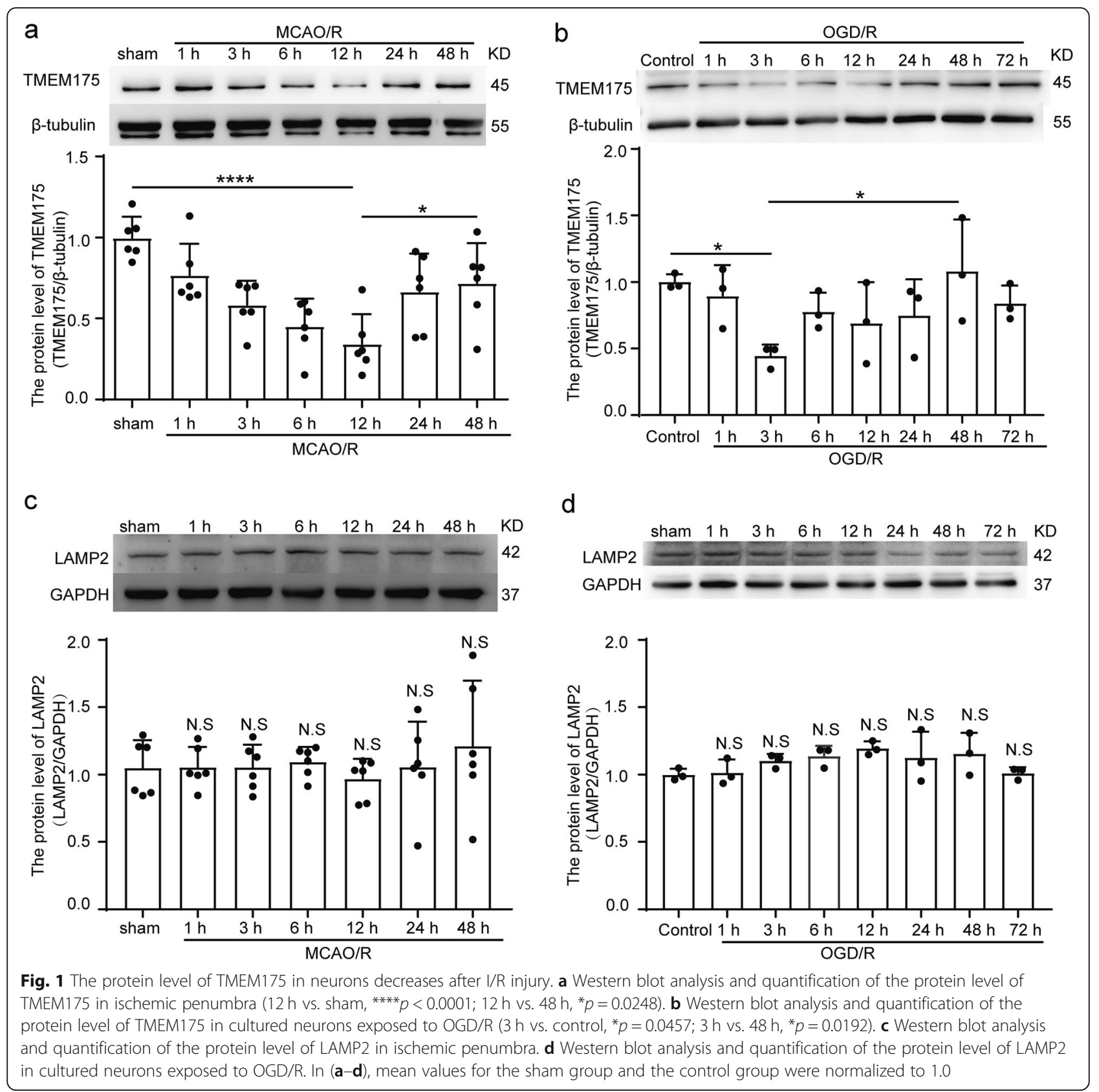

tissues after MCAO/R, we performed TTC and FJC staining. TTC staining showed that TMEM175 overexpression reduced the infarct volume (Fig. 2b). In addition, FJC staining also revealed a rescuing effect of TMEM175 overexpression on neuronal degeneration. (Fig. 2c). In long-term experiments, as shown in Fig. 3a and b, assessments via the Morris water maze revealed that TMEM175 overexpression did not facilitate spatial learning and memory after MCAO/ R. However, TMEM175 upregulation alleviated sensory and motor deficits caused by $\mathrm{MCAO} / \mathrm{R}$, as suggested by results of the rotarod test (Fig. 3c) and adhesive-removal test (Fig. 3d and e). Collectively, these data support that
TMEM175 overexpression improved neuronal death and neurobehavioral deficits after $\mathrm{MCAO} / \mathrm{R}$.

\section{TMEM175 overexpression reduces neuronal injury induced by $O G D / R$ in vitro}

In order to explore the protective role of TMEM175 overexpression under OGD/R conditions, we regulated the protein levels of TMEM175 using over-expression constructs in cultured neurons. To verify the transfection efficiency, we constructed GFP-TMEM175 nonfused overexpressed plasmid. The results showed that the transfection efficiency was stable at about $65 \%$ 


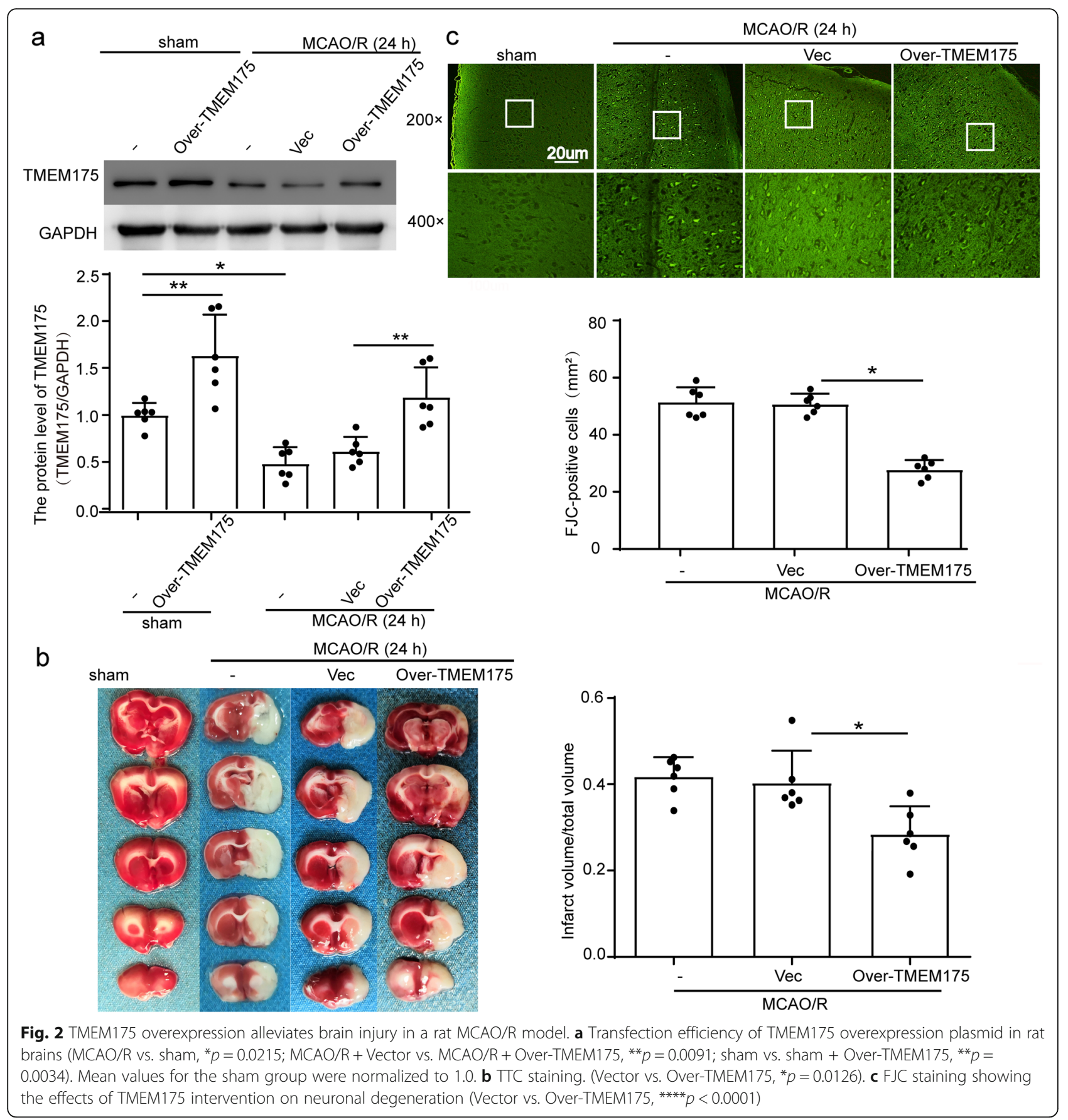

(Supplementary Figure 2). In order to avoid the effect of GFP on the fluorescence detection of Cell-Death/Viability Assays and FJC Staining, our subsequent experiments were carried out based on the transfection of TMEM175 overexpressed plasmid. The results of Western blot analysis showed that, compared with that of the control group, the level of TMEM175 was significantly decreased in the OGD/R group, while over-expression of TMEM175 increased the level of TMEM175. The protein expression level of TMEM175 is normalized by
GAPDH expression. (Fig. 4a). Furthermore, we challenged neuronal cultures for $120 \mathrm{~min}$ with $\mathrm{OGD} / \mathrm{R}$, which is an in-vitro model of ischemia. Four hours later, neuronal death was assessed via Hoechst-33,258 nuclear staining and a live/dead viability assay. As shown in Fig. 4b-e, TMEM175 overexpression reduced the percentage of dead neurons after OGD/R. This result indicates that TMEM175 overexpression increased neuronal resistance against ischemic injury induced by $\mathrm{OGD} / \mathrm{R}$. 


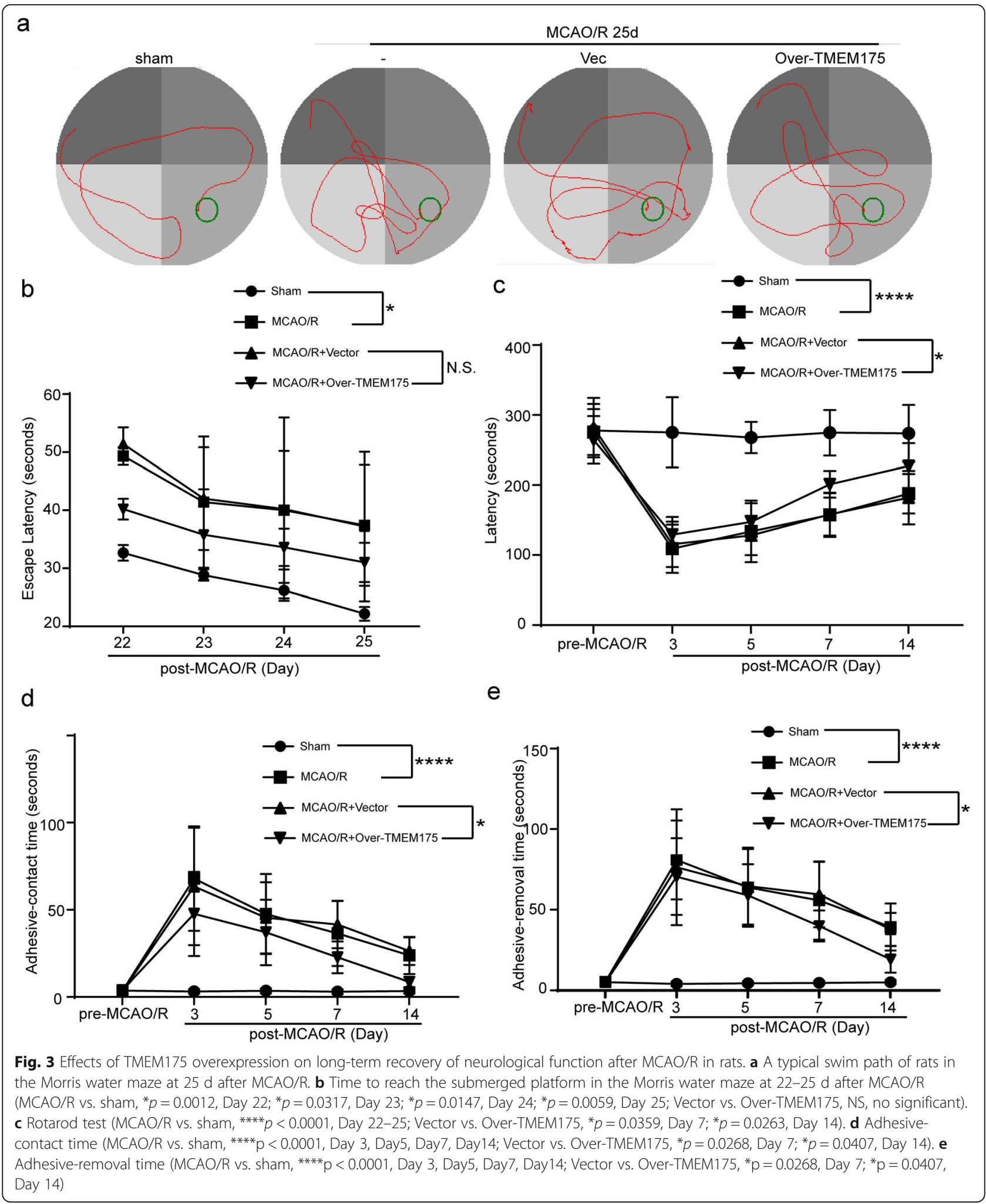

TMEM175 Upregulation increased the clearance of damaged mitochondria after $\mathrm{OGD} / \mathrm{R}$

We used Mito-Sox staining to detect ROS production under different conditions to measure mitochondrial
ROS. As shown in Fig. 5a, ROS levels were significantly increased in the OGD/R group compared with those in the control group. Moreover, TMEM175 overexpression dramatically reduced ROS generation. 


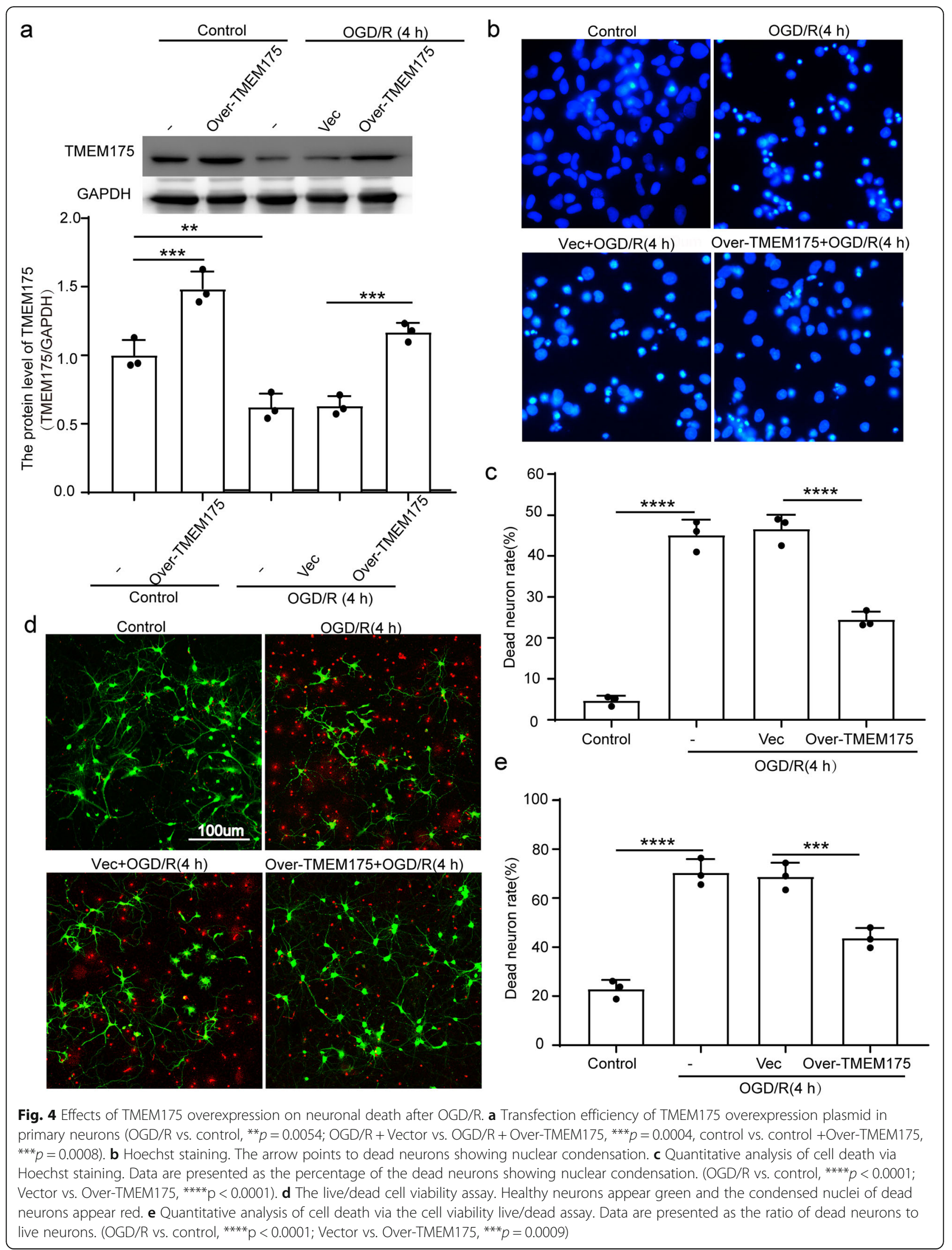




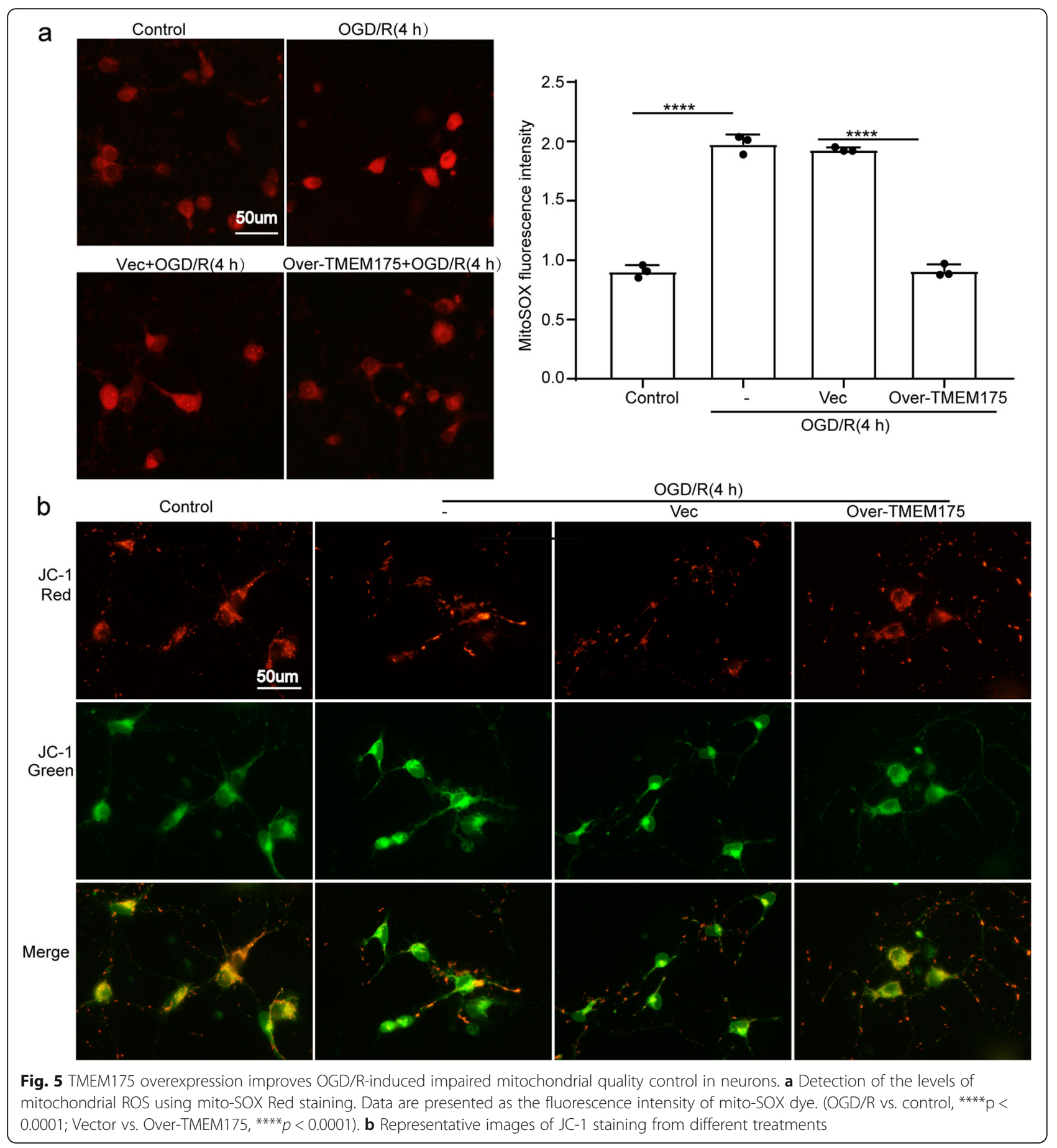

Active mitochondria produce red-shifted fluorescence by readily accumulating JC1 dye in mitochondria compared with that of less active mitochondria that instead emit green fluorescence; this ratio of red/green fluorescence is indicative of mitochondrial activity within a given cell. As shown in Fig. 5b, OGD/R treatment significantly reduced mitochondrial activity compared with that of the control group, as evidenced by the high ratio of red/green JC1 florescence. However, TMEM175 overexpression significantly increased mitochondrial activity following OGD/R, as evidenced by a low red/green JC1 fluorescent ratio compared with that of emptyvector-transfected neurons following OGD/R-treated, indicating a protective role of TMEM175 overexpression. Collectively, these results suggest that mitochondria were damaged in the course of ischemic injury and that TMEM175 upregulation ameliorated this damage. 
TMEM175 deficiency induced by OGD/R decreases Lysosomal catalytic activity in neurons via destabilization of Lysosomal pH

TMEM175 is a $\mathrm{K}^{+}$channel that mediates potassium conductance across lysosomal and endosomal membranes and thus regulates lysosomal membrane potential and $\mathrm{pH}$. We evaluated whether TMEM175 deficiency disrupts autophagic flux by inhibiting lysosomal acidification. AO emits red fluorescence in acidic compartments. It is well recognized that bafilomycin A1 disrupts autophagic flux by inhibiting lysosomal acidification; thus, we used it as a positive control. At $4 \mathrm{~h}$ after OGD/R in DMEM medium, a significant increase in lysosomal $\mathrm{pH}$ was observed. However, compared with that of the bafilomycin A1-treated group, cells after starvation exhibited more bright red fluorescence in the cytoplasm, suggesting that OGD/R affected lysosomal pH (Fig. 6a). To further investigate whether OGD/R affects the hydrolytic function of lysosomes, we measured lysosomal enzymatic activity. As the major lysosomal proteases, the enzymatic activities of CTSB and CTSD were measured. Both CTSB and CTSD activities decreased after OGD/R (Fig. 6b). These results were confirmed by LysoSensor Green staining (Fig. 6c). LysoSensor dyes are acidotropic probes that appear to accumulate in acidic organelles. Thus, LysoSensor reagents exhibit a $\mathrm{pH}$-dependent increase in fluorescent intensity upon acidification. As shown in Fig. 6c, compared with that in the control group, the fluorescent intensity was significantly attenuated in the $\mathrm{OGD} / \mathrm{R}$ group, which was reversed via TMEM175 overexpression. These results suggest that OGD/R impaired the hydrolytic function and/or quantity of lysosomes.

\section{Discussion}

In this study, we demonstrated that cerebral $I / R$ injury down-regulates the protein level of TMEM175 in neurons. TMEM175 deficiency leads to compromised stability of lysosomal $\mathrm{pH}$, affecting the hydrolytic function of lysosomes. Hence, the process by which dysfunctional proteins and organelles, including damaged mitochondria, are degraded by lysosomes is suppressed. Exogenous up-regulation of TMEM175 protein level could reverse the neuronal lysosomal dysfunction after $I / R$ (Fig. 6d).

Cerebral ischemia is a devastating neurological event with high morbidity and mortality, in which a considerable number of mitochondria are damaged following cerebral I/R injury [22]. Recent evidence has demonstrated that autophagy is a lysosome-regulated process for degrading and recycling cellular constituents and is a gatekeeping mechanism for stabilizing cellular homeostasis [23-25]. Mitophagy is a form of selective autophagy that removes damaged mitochondria for mitochondrial quality control in order to maintain homeostasis [25]. Dysfunctional mitophagy results in insufficient removal of damaged mitochondria [26]. It is noteworthy that the impairment of lysosomal catalytic activity results in a decreased capacity of mitophagy $[27,28]$. TMEM175 is a $\mathrm{K}^{+}$ channel located in late endosomes and lysosomes. Depletion of TMEM175 alters lysosomal $\mathrm{pH}$ and decreased the catalytic capacity of lysosomes [10]. Previous reports have established genetic and physiological evidence that TMEM175 is a potential risk factor and candidate therapeutic target for Parkinson's disease and Alzheimer's disease $[7,11]$. However, whether TMEM175 also participates in regulating brain-injury-induced mitophagy has remained unclear.

In the present study, we found that the level of TMEM175 in brain tissues was decreased after ischemic injury, as determined by Western blotting. Analysis of the time course of TMEM175 levels in brain tissues after $\mathrm{MCAO} / \mathrm{R}$ revealed that TMEM175 levels were significantly decreased starting at $3 \mathrm{~h}$ and rebounded at $12 \mathrm{~h}$ after MCAO/R (Fig. 1a). MicroRNAs (miRNAs) are a class of non-coding RNA molecules about 22 nucleotides in size that are involved in the regulation of gene expression at the post-transcriptional level. It is estimated that about $30 \%$ of human gene transcriptome are direct targets of miRNAs, and nearly $90 \%$ of human genes are regulated by miRNAs [29]. Compared with the regulation of gene expression at the transcriptional level, the regulation of gene expression at the posttranscriptional level is more rapid, and the latter may be more suitable for CNS cells to deal with acute stress such as stroke. Based on targetscan prediction, we found that hsa-miR-1268a, hsa-miR-1268band hsa-miR-585-3p have the potential to target TMEM175 mRNA 3'UTR. Studies have shown that hsa-miR-1268a, hsa-miR-1268b and hsa-miR-585-3p levels change in cardiovascular diseases and tumors [30-32], suggesting that their levels are regulated by pathological environment. Therefore, we speculated that the loss of TMEM175 induced by I/R might be regulated by miRNAs such as hsa-miR-1268a, hsa-miR-1268b and hsa-miR-585-3p, which will be further verified in our follow-up work.

Subsequently, we used TMEM175-overexpression plasmids to increase TMEM175 levels in rats prior to the induction of MCAO/R. We found that TMEM175 protein levels in brain tissue were increased at $24 \mathrm{~h}$ after $\mathrm{MCAO} / \mathrm{R}$ and were negatively correlated with the expression of TMEM175 (Fig. 2a). Additionally, we also found that neuronal necrosis in the TMEM175overexpression group was significantly improved compared with that of the control group, and neurobehavioral tests yielded consistent results (Fig. 2 and 3). Therefore, we conclude that TMEM175 deficiency exerts a negative effect on brain tissue in terms of short-time 


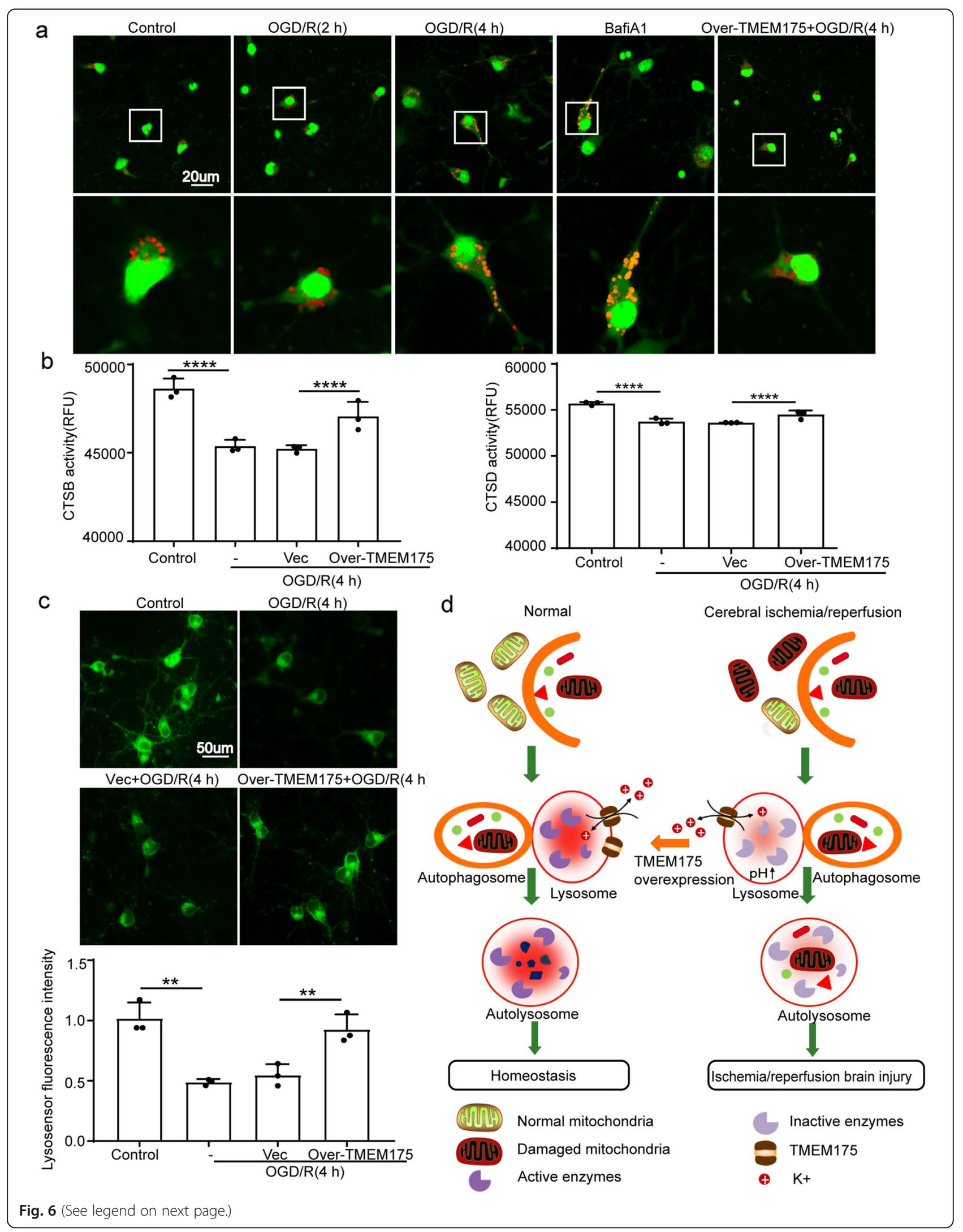


(See figure on previous page.)

Fig. 6 TMEM175 overexpression reverses lysosomal function in neurons exposed to OGD/R. a Fluorescent photographs of acridine-orange (AO) staining of neuronal cultures treated with OGD/R, bafilomycin A1 (100nM), or transfection of TMEM175-overexpression plasmid. b Relative enzymatic activity of CTSB and CTSD in neurons (OGD/R vs. control, ***p $<0.0001$; Vector vs. Over-TMEM175, *** $p<0.0001)$. c Fluorescent photographs of LysoSensor Green staining of neuronal cultures (OGD/R vs. control, ${ }^{* *} p=0.001$; Vector vs. Over-TMEM175, $\left.{ }^{* *} p=0.0082\right)$. d Schematic representation of potential mechanisms of TMEM175 actions in cerebral I/R injury

and long-term brain injury after MCAO/R. Furthermore, TMEM175 deficiency exhibited the same effects in vitro (Fig. 4). We found that damage to neurons induced increased mitochondrial-derived ROS levels, which was reversed by TMEM175 overexpression (Fig. 5a). We also explored the underlying mechanism of TMEM175 deficiency on insufficient removal of damaged mitochondria. The data from acidophilic-dye staining suggested that OGD/R-injury induced unstable lysosomal $\mathrm{pH}$ in primary neurons (Fig. 6a and c).

Existing data points to the relationship between lysosomal $\mathrm{pH}$ and the viability of cells. Bafilomycin A1 is a commonly used compound that inhibits autophagic flux by preventing the acidification of endosomes and lysosomes [33, 34]. In addition, a previous study has shown that Bafilomycin A1 decreased cell viability [26]. Therefore, we could conclude that the $\mathrm{pH}$ change exerted an effect on cell viability of injured cells.

CTSB and CTSD are the most abundant lysosomal cysteine and aspartyl proteases that contribute to the degradation of autolysosomes [35]. The enzymatic function of these enzymes is influenced by the $\mathrm{pH}$ of the surrounding environment. In our present study, the enzymatic activities of CTSB and CTSD were also altered after OGD/R treatment (Fig. 6b), suggesting that TMEM175 deficiency exerts a negative effect on the hydrolytic function of lysosomes following cerebral ischemia with reperfusion. Hence, TMEM175 deficiency may represent a novel late-stage mitophagic inhibitor. Emerging data indicate that mitophagic activation may hold promise as a potential therapeutic strategy against ischemic brain injury [36]. Nevertheless, the mechanisms underlying mitophagy in ischemic neurons are not fully understood. Recent investigations have highlighted roles of PARK2, an E3 ubiquitin ligase, as well as BNIP3L/ NIX, in mitophagy [37-42]. TMEM175 is a novel $\mathrm{K}^{+}$ channel independent of these proteins. It is noteworthy that impaired mitophagy might link TMEM175 deficiency to impairment of mitochondrial clearance. Direct inhibition of autophagy through genetic and pharmacological methods has been shown to decrease mitochondrial oxygen consumption and energy production through the accumulation of dysfunctional mitochondria [43-47]. TMEM175-deficient cells may also be caused by these mechanisms. However, the exact mechanism remains to be elucidated.
Several limitations of our present study should be noted. First, we demonstrated that TMEM175 deficiency inhibited lysosomal function via unstable $\mathrm{pH}$ and decreased protease activity. However, whether the integrity of the lysosome is also compromised by the dysregulation of lysosomal pH requires further investigation. Additionally, although our current study provides evidence that TMEM175 deficiency may disrupt autophagic flux by inhibiting lysosomal acidification, we did not more deeply address whether TMEM175 deficiency affects the fusion of autophagosomes with lysosomes after OGD/R. Second, since we only detected TMEM175 expression within $48 \mathrm{~h}$ after MCAO/R, TMEM175 expression levels in chronic-stage post- $\mathrm{MCAO} / \mathrm{R}$ remain unclear. Third, we only interfered with the expression of TMEM175 using a TMEM175-overexpression plasmid. To reduce the level of TMEM175 expression, a specific siRNA of TMEM175 or knockout of TMEM175 (KO) should be applied in future studies to determine the effect of TMEM175 depletion in I/R injury. Finally, only adult male SD rats are used in our study.

In summary, our present study represents the first to investigate the expression of TMEM175 in MCAO/R rats, and we found that TMEM175 deficiency may be harmful to the recovery of ischemic injury. Furthermore, we found that TMEM175 is associated with lysosomal $\mathrm{pH}$, lysosomal proteolytic activity, and mitochondrial clearance. Therefore, this study lays the foundation for further investigations on the regulatory mechanisms of TMEM175 in the course of autophagy in I/R injury and suggests that TMEM175 may represent a therapeutic target for the amelioration of brain injury after $I / R$ injury.

\section{Supplementary information}

Supplementary information accompanies this paper at https://doi.org/10. 1186/s13041-020-00651-z.

\section{Additional file 1. Supplementary material.}

\section{Abbreviations}

I/R: Ischemia-reperfusion; MCAO/R: Middle-cerebral-artery occlusion/ reperfusion; OGD/R: Oxygen-glucose deprivation/reoxygenation; $A O$ staining: Acridine orange staining; CTSB: Cathepsin-B; CTSD: Cathepsin-D; FJC staining: Fluoro-Jade C (FJC) staining; TTC staining: 2,3,5-Triphenyltetrazolium Chloride staining; ROS: Reactive Oxygen Species

\section{Acknowledgements}




\section{Authors' contributions}

$M Z$ carried out the experiments. HL participated in the design of the study and drafted the manuscript. XX helped to draft the manuscript. HS participated in the acquisition of data. $\mathrm{XL}$ and $\mathrm{YZ}$ carried out analysis and interpretation of data. JW performed the statistical analysis. JN and HL participated in the design of the study. GC revised the manuscript. All authors read and approved the final manuscript.

\section{Funding}

This work was supported by National Key R\&D Program of China. (2018YFC1312600, 2018YFC1312601 and 2017YFC0110304), National Natura Science Foundation of China (81830036, 81771255, 81771254, 81701309), Project of Jiangsu Provincial Medical Innovation Team (CXTDA2017003), Jiangsu Provincial Medical Key Talent grant (ZDRCA2016040), Jiangsu Provincial Medical Youth Talent (QNRC2016728), Suzhou Key Medical Centre (Szzx201501), Scientific Department of Jiangsu Province (BE2017656), Natural Science Foundation of Jiangsu province (BK20170363 and BK20180204), Suzhou Science and Technology (SS2019056), Jiangsu Commission of Health (K2019001), and Gusu health personnel training project (GSWS2019030).

\section{Availability of data and materials}

The datasets generated and/or analysed during the current study are not publicly available due to the confidential policy of our hospital but are available from the corresponding author on reasonable request.

\section{Ethics approval and consent to participate}

All animal experiments are strictly in accordance with the guideline of Soochow University institutional Animal Care and Use Committee.

\section{Consent for publication}

Not applicable.

\section{Competing interests}

The authors declare that they have no competing interests.

\section{Author details}

'Department of Neurology, The First Affiliated Hospital of Soochow University, Suzhou, Jiangsu Province, China. ${ }^{2}$ Department of Neurosurgery \& Brain and Nerve Research Laboratory, The First Affiliated Hospital of Soochow University, Suzhou, Jiangsu Province, China. ${ }^{3}$ Jiangsu Key Laboratory of Medical Optics, Suzhou Institute of Biomedical Engineering and Technology, Chinese Academy of Sciences, Suzhou, China.

\section{Received: 7 May 2020 Accepted: 3 August 2020}

\section{Published online: 15 August 2020}

\section{References}

1. Schaller B, Graf R. Cerebral ischemia and reperfusion: the pathophysiologic concept as a basis for clinical therapy. J Cereb Blood Flow Metab. 2004; 24(4):351-71.

2. Fan $\mathrm{L}$, et al. FasL-PDPK1 pathway promotes the cytotoxicity of CD8(+) T cells during ischemic stroke. Transl Stroke Res. 2020;11(4):747-61.

3. Wang R, et al. Optimized mouse model of embolic MCAO: From cerebral blood flow to neurological outcomes. J Cereb Blood Flow Metab. 2020. https://doi.org/10.1177/0271678X20917625.

4. Dave KR, et al. Activation of protein kinase C delta following cerebral ischemia leads to release of cytochrome C from the mitochondria via bad pathway. PLoS One. 2011;6(7):e22057.

5. Shukla V, et al. Recurrent hypoglycemia exacerbates cerebral ischemic damage in diabetic rats via enhanced post-ischemic mitochondrial dysfunction. Transl Stroke Res. 2019;10(1):78-90.

6. Rajawat YS, Bossis I. Autophagy in aging and in neurodegenerative disorders. Hormones (Athens). 2008;7(1):46-61.

7. Vives-Bauza C, Przedborski S. Mitophagy: the latest problem for Parkinson's disease. Trends Mol Med. 2011;17(3):158-65.

8. Zhao $X$, et al. Elaiophylin, a novel autophagy inhibitor, exerts antitumor activity as a single agent in ovarian cancer cells. Autophagy. 2015;11(10): 1849-63.

9. Cang C, et al. TMEM175 Is an Organelle K(+) Channel Regulating Lysosomal Function. Cell. 2015;162(5):1101-12.
10. Jinn S, et al. TMEM175 deficiency impairs lysosomal and mitochondrial function and increases alpha-synuclein aggregation. Proc Natl Acad Sci U S A. 2017;114(9):2389-94

11. Santos RX, et al. A synergistic dysfunction of mitochondrial fission/fusion dynamics and mitophagy in Alzheimer's disease. J Alzheimers Dis. 2010; 20(Suppl 2):S401-12.

12. Liu Y, et al. Knockdown of Arginyl-tRNA Synthetase attenuates ischemiainduced cerebral cortex injury in rats after middle cerebral artery occlusion. Transl Stroke Res. 2020. https://doi.org/10.1007/s12975-020-00809-w.

13. Shen $\mathrm{H}$, et al. Role for RIP1 in mediating necroptosis in experimental intracerebral hemorrhage model both in vivo and in vitro. Cell Death Dis. 2017;8(3):e2641.

14. Xie R, et al. Alpha-lipoic acid pre- and post-treatments provide protection against in vitro ischemia-reperfusion injury in cerebral endothelial cells via Akt/mTOR signaling. Brain Res. 2012;1482:81-90.

15. Yuan ZF, et al. 10-Hydroxycamptothecin induces apoptosis in human neuroblastoma SMS-KCNR cells through p53, cytochrome $c$ and caspase 3 pathways. Neoplasma. 2016;63(1):72-9.

16. Zhou Z, et al. Sodium butyrate attenuated neuronal apoptosis via GPR41/ Gbetagamma/PI3K/Akt pathway after MCAO in rats. J Cereb Blood Flow Metab. 2020. https://doi.org/10.1177/0271678X20910533.

17. Novorolsky RJ, et al. The cell-permeable mitochondrial calcium uniporter inhibitor Ru265 preserves cortical neuron respiration after lethal oxygen glucose deprivation and reduces hypoxic/ischemic brain injury. J Cereb Blood Flow Metab. 2020:40(6):1172-81.

18. Wang $Z$, et al. Identification of two phosphorylation sites essential for annexin $\mathrm{A} 1$ in blood-brain barrier protection after experimental intracerebral hemorrhage in rats. J Cereb Blood Flow Metab. 2017;37(7): 2509-25

19. Li X, et al. Loss of Ribosomal RACK1 (Receptor for Activated Protein Kinase C 1) Induced by Phosphorylation at T50 Alleviates Cerebral IschemiaReperfusion Injury in Rats. Stroke. 2018. https://doi.org/10.1161/STROKEAHA. 118.022404

20. Zhang L, et al. Quantitative measurement of motor and somatosensory impairments after mild (30 min) and severe (2 h) transient middle cerebral artery occlusion in rats. J Neurol Sci. 2000;174(2):141-6.

21. Zhang $L$, et al. Identification of compound $C A-5 f$ as a novel late-stage autophagy inhibitor with potent anti-tumor effect against non-small cell lung cancer. Autophagy. 2019;15(3):391-406.

22. White $B C$, et al. Brain ischemia and reperfusion: molecular mechanisms of neuronal injury. J Neurol Sci. 2000;179(S 1-2):1-33.

23. Yu S, et al. Inhibition of mitochondrial calcium uniporter protects neurocytes from ischemia/reperfusion injury via the inhibition of excessive mitophagy. Neurosci Lett. 2016;628:24-9.

24. Zuo W, et al. Mitochondria autophagy is induced after hypoxic/ischemic stress in a Drp1 dependent manner: the role of inhibition of Drp1 in ischemic brain damage. Neuropharmacology. 2014;86:103-15.

25. Li Q, et al. Rapamycin attenuates mitochondrial dysfunction via activation of mitophagy in experimental ischemic stroke. Biochem Biophys Res Commun. 2014;444(2):182-8.

26. Zhang $X$, et al. Cerebral ischemia-reperfusion-induced autophagy protects against neuronal injury by mitochondrial clearance. Autophagy. 2013;9(9): 1321-33.

27. Hara T, et al. Suppression of basal autophagy in neural cells causes neurodegenerative disease in mice. Nature. 2006:441(7095):885-9.

28. Komatsu M, et al. Essential role for autophagy protein Atg7 in the maintenance of axonal homeostasis and the prevention of axonal degeneration. Proc Natl Acad Sci U S A. 2007;104(36):14489-94.

29. Ponomarev ED, Veremeyko T, Weiner HL. MicroRNAs are universal regulators of differentiation, activation, and polarization of microglia and macrophages in normal and diseased CNS. Glia. 2013;61(1):91-103.

30. Gao F, et al. SLC35E3 identified as a target of novel-m1061-5p via microRNA profiling of patients with cardiovascular disease. Mol Med Rep. 2018;17(4): 5159-67.

31. Yaghoobi H, et al. EBST: An Evolutionary Multi-Objective Optimization Based Tool for Discovering Potential Biomarkers in Ovarian Cancer. IEEE/ ACM Trans Comput Biol Bioinform. 2020. https://doi.org/10.1109/TCBB. 2020.2993150

32. Ma Y, et al. Plasma microRNA alterations between EGFR-activating mutational NSCLC patients with and without primary resistance to TKI. Oncotarget. 2017:8(51):88529-36. 
33. Yamamoto A, et al. Bafilomycin A1 prevents maturation of autophagic vacuoles by inhibiting fusion between autophagosomes and lysosomes in rat hepatoma cell line, H-4-II-E cells. Cell Struct Funct. 1998;23(1):33-42.

34. Klionsky DJ, et al. Does bafilomycin A1 block the fusion of autophagosomes with lysosomes? Autophagy. 2008;4(7):849-50.

35. Tatti $\mathrm{M}$, et al. Cathepsin-mediated regulation of autophagy in saposin $\mathrm{C}$ deficiency. Autophagy. 2013;9(2):241-3.

36. Yuan $Y$, et al. Regulation of mitophagy in ischemic brain injury. Neurosci Bull. 2015;31(4):395-406.

37. Huang C, et al. Preconditioning involves selective mitophagy mediated by Parkin and p62/SQSTM1. PLoS One. 2011;6(6):e20975.

38. Zhang $X$, et al. Endoplasmic reticulum stress induced by tunicamycin and thapsigargin protects against transient ischemic brain injury: involvement of PARK2-dependent mitophagy. Autophagy. 2014;10(10):1801-13.

39. Allen GF, et al. Loss of iron triggers PINK1/Parkin-independent mitophagy. EMBO Rep. 2013;14(12):1127-35.

40. Sowter HM, et al. HIF-1-dependent regulation of hypoxic induction of the cell death factors BNIP3 and NIX in human tumors. Cancer Res. 2001;61(18): 6669-73.

41. Birse-Archbold JL, et al. Differential profile of nix upregulation and translocation during hypoxia/ischaemia in vivo versus in vitro. J Cereb Blood Flow Metab. 2005:25(10):1356-65.

42. Bas-Orth C, et al. The mitochondrial calcium uniporter is crucial for the generation of fast cortical network rhythms. J Cereb Blood Flow Metab. 2019. https://doi.org/10.1177/0271678X19887777.

43. Guo JY, et al. Activated Ras requires autophagy to maintain oxidative metabolism and tumorigenesis. Genes Dev. 2011;25(5):460-70

44. Yang $\mathrm{S}$, et al. Pancreatic cancers require autophagy for tumor growth. Genes Dev. 2011;25(7):717-29.

45. Borkowska J, et al. Tuberous sclerosis complex: tumors and tumorigenesis. Int J Dermatol. 2011;50(1):13-20.

46. Liang $X$, et al. Inhibiting systemic autophagy during interleukin 2 immunotherapy promotes long-term tumor regression. Cancer Res. 2012; 72(11):2791-801.

47. Zhang Y, et al. Activation of Sigma-1 receptor enhanced Pericyte survival via the interplay between apoptosis and autophagy: implications for bloodbrain barrier integrity in stroke. Transl Stroke Res. 2020;11(2):267-87.

\section{Publisher's Note}

Springer Nature remains neutral with regard to jurisdictional claims in published maps and institutional affiliations.

Ready to submit your research? Choose BMC and benefit from:

- fast, convenient online submission

- thorough peer review by experienced researchers in your field

- rapid publication on acceptance

- support for research data, including large and complex data types

- gold Open Access which fosters wider collaboration and increased citations

- maximum visibility for your research: over $100 \mathrm{M}$ website views per year

At $\mathrm{BMC}$, research is always in progress.

Learn more biomedcentral.com/submissions 